\title{
Estudo da estabilidade térmica e propriedades mecânicas de nanocompósitos de poliamida 6,6 com nanofibras de celulose
}

\author{
Daiane Benaducci ${ }^{1}$, Marcia Cristina Branciforti ${ }^{1}$
}

\author{
${ }^{1}$ Departamento de Engenharia de Materiais, Escola de Engenharia de São Carlos, Universidade de São Paulo \\ CEP:15566-590 São Carlos, SP. \\ e-mail: daianebenaducci@yahoo.com.br; marciacb@sc.usp.br
}

\begin{abstract}
RESUMO
Neste estudo, a estabilidade térmica e as propriedades mecânicas da poliamida 6,6 (PA) e de seus nanocompósitos com nanofibras de celulose (NFC) foram investigadas. Objetivando a dispersão uniforme da NFC na PA e o processamento a altas temperaturas, os materiais foram inicialmente pré-misturados em solução e posteriormente processados por extrusão e moldagem por injeção. Os resultados obtidos por análise termogravimétrica confirmaram a maior estabilidade térmica das amostras sem NFC em relação as amostras com NFC. A pré-mistura e o processamento influenciaram na estabilidade térmica das amostras. Os resultados obtidos por ensaio de tração revelaram que a adição de $1 \%$ de NFC levou a um aumento médio de $16 \%$ no módulo elástico em relação a PA pura. A pré-mistura e a incorporação das NFC's não prejudicaram o módulo elástico e a resistência máxima à tração, podendo indicar uma alternativa de uso para poliamida 6,6 , dependendo da aplicação final do material.
\end{abstract}

Palavras-chave: Nanofibras de celulose, poliamida 6,6, nanocompósitos estabilidade térmica, propriedades mecânicas.

\begin{abstract}
In this study, the thermal stability and mechanical properties of polyamide 6.6 (PA) and their nanocomposites with cellulose nanofibers (CNF) were investigated. In order to have uniform dispersion of CNF in the PA and the processing at high temperatures, the materials were initially premixed and further processed by extrusion and injection molding. The results obtained by thermogravimetric analysis confirmed the lower thermal stability of the samples without CNF in comparison to the samples with CNF. The premix and the processing influence on the thermal stability of the samples. The results obtained by tensile test showed the addition of $1 \% \mathrm{CNF}$ leads to an average increase of $16 \%$ in the elastic modulus in comparison to the neat PA. The premix and the incorporation of CNF did not impair the elastic modulus and maximum tensile strength, and may indicate an alternative use for PA, depending on the final application of the material.
\end{abstract}

Keywords: Cellulose nanofibers, polyamide 6.6, nanocomposites, thermal stability, mechanical properties.

\section{INTRODUÇÃO}

O estudo das nanofibras de celulose (NFC) como fase de reforço em compósitos poliméricos começou há cerca de 20 anos e desde essa época os pesquisadores tem se dedicado ao isolamento, caracterização e aplicação desses materiais altamente purificados. As NFC's são normalmente produzidas pelo processo de polpação, no qual ocorre uma ruptura das ligações presentes na estrutura da madeira, possibilitando a individualização das fibras, seguido pelo processo de refino da celulose, onde a suspensão de fibras diluídas é sujeita a tensões cíclicas repetidas, produzindo a fibrilação externa das mesmas, e a última etapa consiste no processo de homogeneização, no qual a suspensão de fibras de celulose é bombeada a alta pressão através de um conjunto de válvulas que abrem e fecham de forma rápida e sucessiva, fazendo com que as fibras sofram uma grande queda de pressão, com forças de cisalhamento e impacto, proporcionando assim um elevado grau de microfibrilação das fibras de celulose, resultando nas NFC [1].

Um dos problemas relacionados aos materiais lignocelulósicos é a temperatura de processamento dos compósitos ser limitada a faixas em torno de $200^{\circ} \mathrm{C}$, devido ao fato da hemicelulose presente nas fibras começar a degradar próximo de $230^{\circ} \mathrm{C}$. Assim, a temperatura de fusão dos materiais plásticos selecionados não 
deve ser superior a $200^{\circ} \mathrm{C}$, restringindo a utilização de termoplásticos de engenharia como a poliamida. Por outro lado, os plásticos de engenharia são capazes de oferecer elevada resistência química, mecânica e térmica em setores de alta tecnologia, como os setores de eletroeletrônicos e o automotivo. Esses plásticos são utilizados progressivamente como alternativa aos metais, vidros e outros materiais, resultando em significativa redução no peso do material final e aumento na flexibilidade de design dos mesmos, o que possibilita projetos diferenciados. No caso dos automóveis a redução de peso faz com que a emissão de $\mathrm{CO}_{2}$ seja reduzida, promovendo assim a sustentabilidade. Uma vantagem adicional para as montadoras no setor automotivo é o aumento na vida útil das peças, sinônimo de prazos de garantia maiores, e ainda pelas melhorias de produtividade, conquistadas com a eliminação de pinturas ou redução de operações secundárias como soldagem, colagem, fixações, entre outras [2].

Nesse contexto, pesquisadores buscam soluções em alternativas relacionadas ao método de processamento, uso de aditivos e modificações na estrutura do material celulósico para o desenvolvimento de novos compósitos. Estudos de análise termogravimétrica dos componentes poliméricos da madeira mostram que o componente celulósico purificado é termicamente mais estável do que outros componentes poliméricos da madeira. Devido à maior estabilidade térmica em comparação com demais fibras de madeira, essas celuloses purificadas podem ser utilizadas como reforço de termoplásticos de engenharia [3,4].

Em paralelo a esse cenário, pesquisadores têm focado seus trabalhos no processamento de nanocompósitos, devido aos fortes efeitos de reforço a uma fração de volume baixo [5-9]. Na nanoescala surgem novos fenômenos que não aparecem na macroescala. Esta alteração de comportamento está relacionada com as forças naturais fundamentais que mudam de importância quando a escala é reduzida [10]. As vantagens de materiais nanocompósitos, quando comparados com os compósitos convencionais são as suas excelentes propriedades térmicas, mecânicas e de barreira, bem como a sua melhor capacidade de reciclagem, sua transparência e menor densidade $[1,7,11]$. Essas vantagens se devem ao fato das cargas de tamanho nanométrico apresentarem uma área de superfície maior em comparação com enchimentos tradicionais, promovendo maior interação com a matriz e possivelmente uma alteração mais significativa nas propriedades físico-químicas do nanocompósito final. Além do fato de apenas pequenas quantidades serem suficientes para garantirem propriedades finais diferenciadas aos nanocompósitos.

Apesar das propriedades atraentes dos reforços celulósicos, eles são utilizados de forma limitada na prática industrial, devido às dificuldades associadas à compatibilidade e às interações superficiais. Esses reforços possuem natureza polar e hidrofílica enquanto que a maior parte dos polímeros termoplásticos mais comumente utilizados possui características não-polares, prejudicando a obtenção de níveis aceitáveis de dispersão [3,12]. Por isso a importância na escolha de materiais, dos métodos de fabricação, dos parâmetros de processamento e do tratamento da superfície das fibras ou do uso de aditivos. Essas escolhas podem afetar também a adesão na interface fibra/matriz.

A intensidade da interação na interface é fundamental para garantir as propriedades mecânicas de um compósito. Se o nível de adesão entre o material de reforço e a matriz não é suficiente, uma via de difusão pode pré-existir ou pode ser criada sob solicitação mecânica [3]. Uma boa interação entre a matriz e o reforço pode ser garantida se o material de reforço hidrofílico estiver completamente encapsulado numa matriz de polímero hidrofóbico ou se ambos possuírem a mesma natureza polar.

Nesse contexto, as poliamidas se tornam boas candidatas como uma matriz compatível para as NFC. As poliamidas alifáticas são muito conhecidas na indústria de plásticos de engenharia e podem ser produzidas comercialmente por condensação de diaminas com os ácidos dibásicos, pela auto-condensação de um aminoácido ou pela polimerização com abertura de anel de uma caprolactana [13]. Apesar do grande interesse tecnológico nas poliamidas, apenas alguns estudos de compósitos poliméricos de poliamidas 6 e 6,6/celulose foram publicados $[4,8,9,14]$. O principal desafio no desenvolvimento desses compósitos está relacionado com a baixa estabilidade térmica dos materiais celulósicos e a relativa alta temperatura necessária no processamento convencional das poliamidas. Kaziltas e colaboradores $[4,14]$ estudaram extensivamente a aplicação de celulose microcristalina como agente de reforço em poliamida 6 e 6,6 no estado fundido, devido ao fato da celulose microcristalina apresentar maior estabilidade térmica quando comparada com outras estruturas nanocelulósicas. Qua e Hornsby [8] obtiveram filmes de compósitos de poliamida 6 reforçados com nanocristais de celulose via solução casting. No estudo de Corrêa e colaboradores [9], nanocristais de celulose foram revestidos com poliamida 6 , resultando em um material com maior estabilidade térmica, o qual foi utilizado na obtenção de nanocompósitos com poliamida 6 processados no estado fundido a altas temperaturas.

No presente estudo foram preparados nanocompósitos a base de NFC e poliamida 6,6. Foi utilizada a poliamida 6,6 comercial produzida pela Rhodia e a NFC também comercial fabricada na Universidade de Toronto. $\mathrm{O}$ estudo teve como objetivos a caracterização das propriedades térmicas e mecânicas da poliamida bem como de seus nanocompósitos reforçados com as NFC. Buscou-se uma solução alternativa relacionada 
ao método de processamento, a pré-mistura, visando a possibilidade de processamento a altas temperaturas e a produção de nanocompósitos com dispersão homogênea da NFC na matriz polimérica.

\section{MATERIAIS E MÉTODOS}

\subsection{Materiais}

A poliamida 6,6 (PA) foi cedida pela empresa Rhodia Poliamida e Especialidades Ltda do Brasil e possui massa molar média $\left(\mathrm{M}_{\mathrm{w}}\right)$ de $20.000 \mathrm{~g} / \mathrm{mol}$, densidade de $1,14 \mathrm{~g} / \mathrm{cm}^{3}$, temperatura de fusão cristalina $\left(\mathrm{T}_{\mathrm{m}}\right)$ na faixa de $255-265^{\circ} \mathrm{C}$, temperatura de transição vítrea $\left(\mathrm{T}_{\mathrm{g}}\right)$ na faixa de $55-65$ e grau de cristalinidade $\left(\mathrm{X}_{\mathrm{c}}\right)$ entre $20-40 \%$. As nanofibras de celulose (NFC) foram gentilmente cedidas pelo Centro de Biocompósitos e Processamento de Biomateriais da Universidade de Toronto, Canadá, são compostas por $84,6 \pm 4,4 \% \alpha$-celulose, $6,0 \pm 1,1 \%$ de hemicelulose e de $9,4 \pm 0,8 \%$ de lignina, possuem valores médios de diâmetro (D) de 10-25 $\mathrm{nm}$ e comprimento (L) de (800-1100 nm), o que corresponde a uma razão de aspecto (L/D) de 110-30 e grau de cristalinidade de aproximadamente $73 \%$ [15-17]. O ácido fórmico com grau de pureza de $85 \%$ foi adquirido da empresa Synth.

\subsection{Método de preparação dos nanocompósitos}

\subsubsection{Pré-mistura em solução}

Foi preparada solução de $24 \%$ em massa do polímero original (PA) dissolvido no ácido fórmico a $45^{\circ} \mathrm{C}$ sob agitação constante. Posteriormente as nanofibras de celulose (NFC, $12 \%$ em massa) foram dispersas no ácido fórmico e a suspensão obtida foi adicionada à solução polimérica, mantendo as proporções de 0,1 e $2 \% \mathrm{em}$ massa de NFC. Em seguida as misturas foram adicionadas a um não-solvente, água deionizada, em excesso e os materiais precipitados (PA-P0, PA-P1 e PA-P2, respectivamente) foram secos em estufa à vácuo a $100^{\circ} \mathrm{C}$ por $48 \mathrm{~h}$.

\subsubsection{Extrusão e moldagem por injeção}

Os materiais pré-misturados (PA-P0, PA-P1 e PA-P2) e a matriz de PA pura (PA-0) foram processados na miniextrusora e miniinjetora da DSM Xplore. A extrusora opera com dupla-rosca corrotantes e cônicas e possui 3 zonas de aquecimento. Como parâmetros de processo de extrusão foram usados: rotação de rosca de 100RPM e temperaturas nas zonas de aquecimento de $280^{\circ} \mathrm{C} / 280^{\circ} \mathrm{C} / 280^{\circ} \mathrm{C}$. A miniinjetora possui capacidade para $15 \mathrm{~g}$ de material e pode operar com pressão máxima de $16 \mathrm{bar}$. Como parâmetros da moldagem por injeção foram usados: temperatura de injeção de $280^{\circ} \mathrm{C}$, pressão de injeção de $4 \mathrm{bar}$, tempo de injeção de $5 \mathrm{~s}$, tempo de recalque $2 \mathrm{~s}$, temperatura do molde de $40^{\circ} \mathrm{C}$ e tempo de resfriamento no molde de $60 \mathrm{~s}$. Os corpos de prova para ensaios mecânico de tração foram obtidos segundo a norma ASTM D1708. A Tabela 1 apresenta a composição dos materiais estudados.

Tabela 1: Composição dos materiais estudados.

\begin{tabular}{l|l|l}
\hline AMOSTRA & $\begin{array}{l}\text { PA } \\
\text { (\% em massa) }\end{array}$ & $\begin{array}{l}\text { NFC } \\
\text { (\% em massa) }\end{array}$ \\
\hline PA & 100 & - \\
\hline PA-0 & 100 & - \\
\hline PA-P0 & 100 & - \\
\hline PA-P1 & 99 & 1 \\
\hline PA-P2 & 98 & 2 \\
\hline
\end{tabular}

\subsection{Técnicas de caracterização}

A estabilidade térmica dos materiais foi estudada por análise termogravimétrica (TGA), a qual foi realizada no equipamento da PerkinElmer Pyris 1TG. Amostras com massa de aproximadamente 7,0 mg foram colocadas em porta-amostra de platina e as corridas foram realizadas na faixa de $25-700^{\circ} \mathrm{C}$, com taxa de aquecimento de $10^{\circ} \mathrm{C} / \mathrm{min}$ em atmosfera de nitrogênio com fluxo de $20 \mathrm{~mL} / \mathrm{min}$.

As propriedades mecânicas das amostras foram avaliadas por ensaios de tração. Os corpos de prova obtidos por moldagem via injeção segundo a norma ASTM D1708 foram acondicionados em ambiente com umidade relativa de $45 \pm 2 \%$ por $48 \mathrm{~h}$. Os ensaios foram realizados em máquina de ensaios universal EMIC 
DL3000 segundo a norma ASTM D638. A velocidade de separação das garras utilizada foi de $5 \mathrm{~mm} / \mathrm{min} \mathrm{e}$ célula de carga de $500 \mathrm{kgf}$ com o objetivo de determinar o módulo elástico $(\mathrm{E})$, resistência à tração $\left(\sigma_{\max }\right)$ e deformação na ruptura $(\varepsilon)$.

\section{RESULTADOS E DISCUSSÕES}

A Figura 1 apresenta os termogramas da análise termogravimétrica (TG e dTG) para a NFC pura. A primeira queda da curva em $100^{\circ} \mathrm{C}$ indica a primeira perda de massa, correspondente à perda de água da amostra. A curva manteve-se praticamente constante até aproximadamente $295^{\circ} \mathrm{C}$ e a perda de massa a partir da temperatura ambiente até esse ponto foi em torno de $10 \%$. A decomposição térmica da NFC inicia-se em torno de $295^{\circ} \mathrm{C}\left(\mathrm{T}_{\mathrm{i}}\right)$, correspondendo à decomposição da celulose, e segue até praticamente massa constante em torno de $430^{\circ} \mathrm{C}\left(\mathrm{T}_{\mathrm{f}}\right)$. O teor de resíduos foi aproximadamente $6,5 \%$. A curva de dTG mostra a máxima velocidade de perda mássica da $\mathrm{NFC}$ em $390^{\circ} \mathrm{C}\left(\mathrm{T}_{\text {deg.máx. }}\right)$. Esta melhor estabilidade térmica encontrada em comparação com outras fibras naturais é devido também ao processo de obtenção das NFC, que elimina uma grande quantidade da lignina e hemicelulose. Esses resultados são consistentes com os relatados na literatura [15, 16, 18].

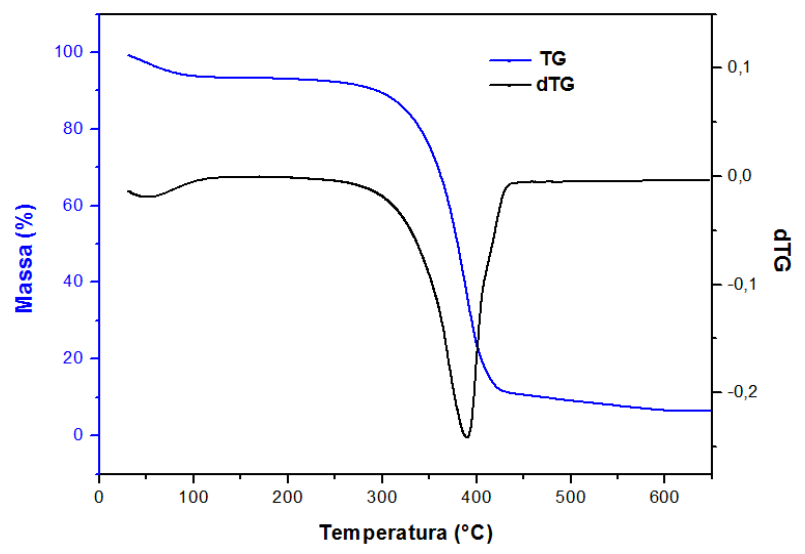

Figura 1: Termogramas TG e dTG da NFC.

A Figura 2 apresenta as curvas de análise termogravimétrica (TG e dTG) para o polímero puro, PA. Da temperatura ambiente até aproximadamente $370^{\circ} \mathrm{C}$ observa-se a perda de massa de apenas $2 \%$, correspondendo à perda de umidade. A decomposição térmica inicia-se em torno de $380^{\circ} \mathrm{C}\left(\mathrm{T}_{\mathrm{i}}\right)$, indo praticamente com massa constante até aproximadamente $700^{\circ} \mathrm{C}$. O teor de resíduos em $600^{\circ} \mathrm{C}$ foi aproximadamente $0,4 \%$, o qual pode estar associado aos resíduos oriundos do processo de síntese. A perda de massa possui taxa máxima em $468^{\circ} \mathrm{C}\left(\mathrm{T}_{\text {deg.máx. }}\right)$ na curva dTG. Esses resultados encontrados são consistentes com os relatados na literatura [19] e são listados na Tabela 2 para facilitar a comparação.

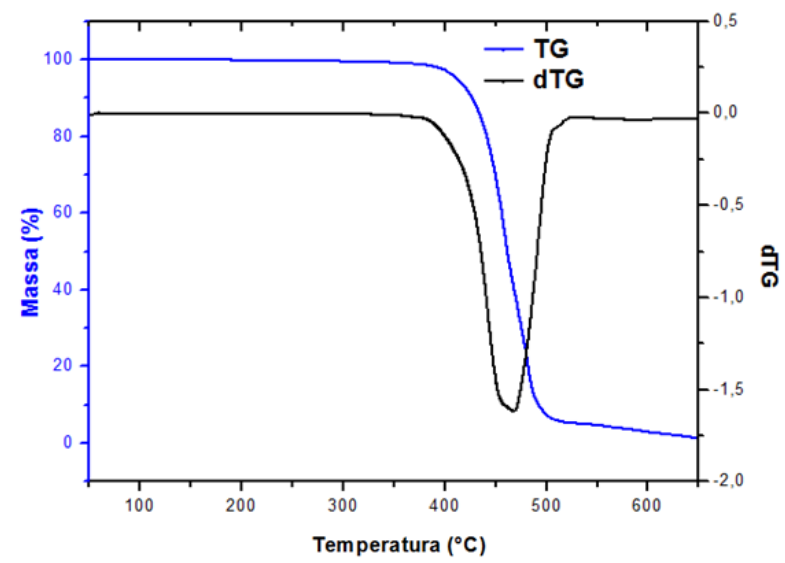

Figura 2: Termogramas TG e dTG do polímero puro PA. 
Todos os corpos de prova moldados por injeção apresentaram aspecto homogêneo com uma leve coloração amarelada após o processamento em relação aos pellets da resina original. Em geral, no caso das amostras sem NFC, o amarelamento é possivelmente devido à degradação pirolítica comumente observada em poliamidas, no caso das amostras com NFC, essa coloração pode ser indicação da decomposição térmica da NFC, pois o processamento foi necessariamente dado no limite de temperatura de fusão da $\mathrm{PA}\left(280^{\circ} \mathrm{C}\right) \mathrm{e}$ muito próximo a temperatura de início de degradação térmica das $\mathrm{NFC}\left(\mathrm{T}_{\mathrm{i}}=295^{\circ} \mathrm{C}\right)$.

Nas figuras 3 (a) e (b) são apresentadas as curvas de TG e dTG, respectivamente, para as amostras PA-0, PA-P0, PA-P1 e PA-P2. Para facilitar a comparação, os dados obtidos a partir das análises foram sumarizados na Tabela 2.
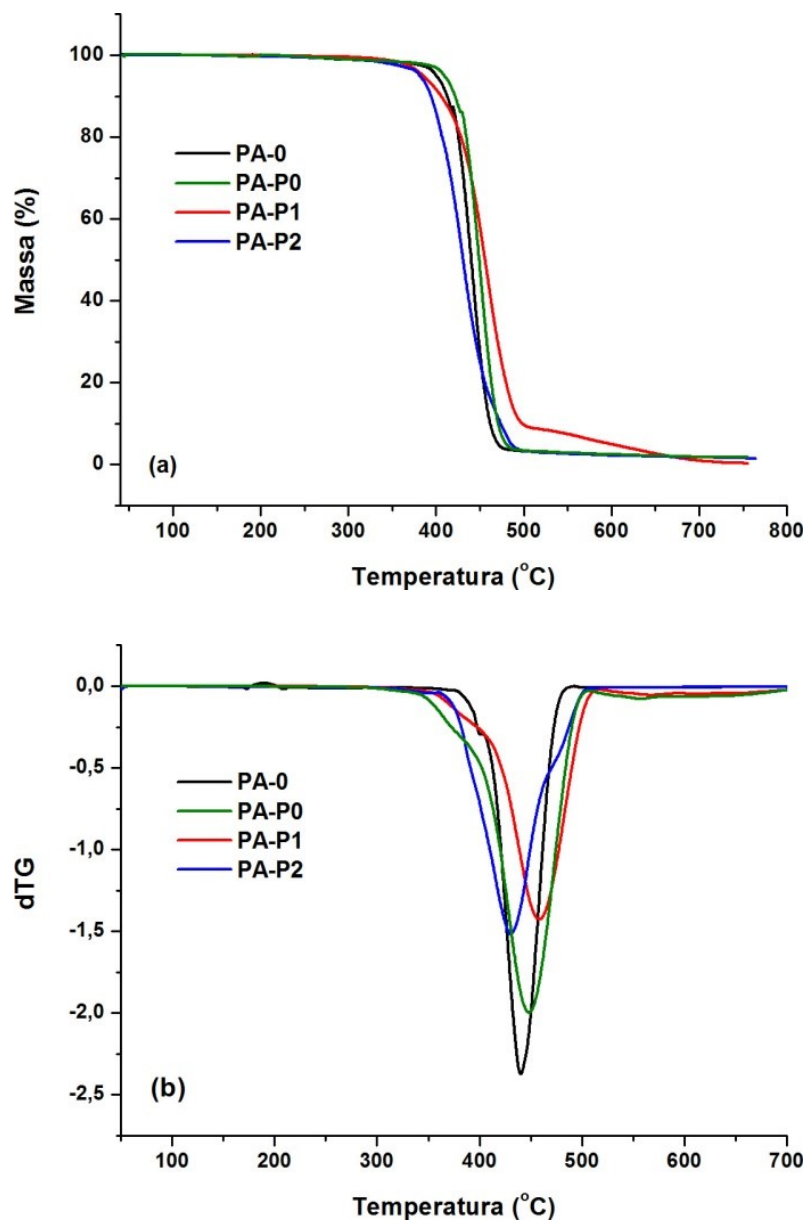

Figura 3: Termogramas (a) TG e (b) dTG das amostras PA-0, PA-P0, PA-P1 e PA-P2.

$\mathrm{Na}$ curva de TG da Figura 3(a) observa-se que até $330^{\circ} \mathrm{C}$ todas as amostras apresentam estabilidade térmica semelhante e perda de massa em voláteis de apenas $0,9 \%$, em média. A incorporação das NFC influenciou a $T_{i}$, fazendo com que os nanocompósitos apresentassem estabilidade térmica inferior se comparados às amostras de polímeros puros (PA-0 e PA-P0), isso se deve ao fato da NFC se decompor em temperatura inferior $\left(\mathrm{T}_{\mathrm{i}}=295^{\circ} \mathrm{C}\right.$, Figura 1) a da PA original $\left(\mathrm{T}_{\mathrm{i}}=380^{\circ} \mathrm{C}\right.$, Figura 2). A decomposição dos nanocompósitos pré-misturados com NFC inicia-se em torno de $348^{\circ} \mathrm{C}\left(\mathrm{T}_{\mathrm{i}}\right)$ para a amostra $\mathrm{PA}-\mathrm{P} 1$ e $344^{\circ} \mathrm{C}\left(\mathrm{T}_{\mathrm{i}}\right)$ para a amostra PA-P2, e para a amostra sem NFC (PA-P0) em $370^{\circ} \mathrm{C}\left(\mathrm{T}_{\mathrm{i}}\right)$. O fato da decomposição térmica da amostra de PA-0 ter iniciado abaixo da decomposição térmica encontrada para a resina original, amostra PA, (Figura 2) indica a influência do processamento (extrusão e injeção) na estabilidade térmica dos materiais. Por outro lado, a pré-mistura não comprometeu negativamente a estabilidade térmica da PA, pois não influenciou a temperatura de decomposição inicial $\left(\mathrm{T}_{\mathrm{i}}\right)$, e resultou em aumento na temperatura final de degradação $\left(\mathrm{T}_{\mathrm{f}}\right)$ e na temperatura de máxima taxa de degradação térmica (Tdeg.máx.), como pode ser evidenciado pelos dados da Tabela 2. Os picos na curva dTG (Figura 3(b)) das amostras processadas encontram-se entre $430^{\circ} \mathrm{C}$ e $460^{\circ} \mathrm{C}$. As amostras apresentaram teor de resíduos em $700^{\circ} \mathrm{C}$ inferior a $2 \%$. 
Tabela 2: Resultados das análises de TGA das amostras. Temperatura inicial de degradação $\left(\mathrm{T}_{\mathrm{i}}\right)$, temperatura final de degradação $\left(\mathrm{T}_{\mathrm{f}}\right)$ e temperatura de máxima taxa de degradação térmica $\left(\mathrm{T}_{\text {deg.máx. }}\right)$.

\begin{tabular}{l|l|l|l|l|l}
\hline AMOSTRA & $\mathbf{T}_{\mathbf{i}}\left({ }^{\circ} \mathbf{C}\right)$ & $\mathbf{T}_{\mathbf{f}}\left({ }^{\circ} \mathbf{C}\right)$ & RESÍDUOS (\%) & VOLÁTEIS (\%) & $\mathbf{T}_{\text {deg. máx. }\left({ }^{\circ} \mathbf{C}\right)}$ \\
\hline PA & 380 & 530 & 2,0 & 0,8 & 468 \\
\hline PA-0 & 370 & 500 & 1,0 & 0,9 & 440 \\
\hline PA-P0 & 370 & 510 & 1,0 & 0,8 & 450 \\
\hline PA-P1 & 348 & 530 & 0,5 & 0,8 & 460 \\
\hline PA-P2 & 344 & 510 & 1,0 & 0,9 & 430 \\
\hline
\end{tabular}

Corrêa e colaboradores [9] demonstraram que concentrado de nanocristais de celulose revestidos com poliamida 6 apresentou elevada estabilidade a degradação térmica. Nanocristais de celulose apresentaram temperatura inicial de degradação térmica $\left(\mathrm{T}_{\mathrm{i}}\right)$ de $180^{\circ} \mathrm{C}$ e após serem revestidos com uma fina camada de poliamida 6 passaram a apresentaram $\mathrm{T}_{\mathrm{i}}$ de $280{ }^{\circ} \mathrm{C}$. Esse aumento significativo na estabilidade térmica do concentrado permitiu seu uso no processamento de nanocompósitos com poliamida 6 a temperatura elevada apropriada.

Na Figura 4 são apresentadas as curvas tensão-deformação obtidas através dos ensaios de tração das amostras processadas PA-0, PA-P0, PA-P1 e PA-P2. Foram realizados cinco ensaios para cada amostra e com os valores obtidos foram calculadas as médias aritméticas e as estimativas de desvio padrão. Os valores médios do módulo elástico $(\mathrm{E})$, resistência à tração $\left(\sigma_{\max }\right)$ e deformação na ruptura $(\varepsilon)$, calculados a partir das curvas tensão-deformação estão apresentados na Tabela 3.

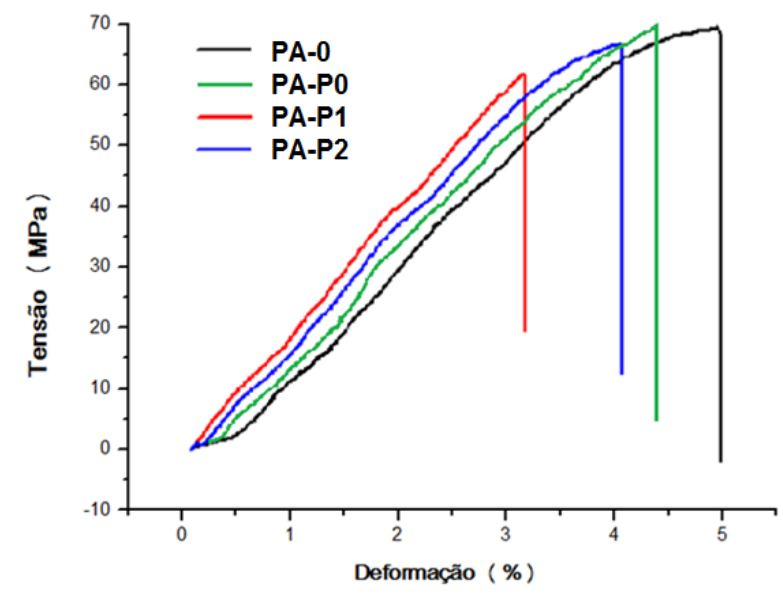

Figura 4: Exemplos de curvas tensão-deformação das amostras PA-0, PA-P0, PA-P1 e PA-P2.

Os resultados apresentados na Figura 4 evidenciam que a etapa de pré-mistura não prejudicou o módulo elástico e a resistência a tração quando comparadas as amostras PA-0 e PA-P0. Entretanto a amostra pré-misturada apresentou ductilidade inferior a da amostra não pré-misturada. O módulo elástico da amostra PA-P0 foi de 1,9 $\pm 0,1 \mathrm{GPa}$ e aumentou para 2,2 $\pm 0,1 \mathrm{GPa}$ com adição de apenas 1\% de NFC (amostra PAP1), correspondendo a um aumento de $16 \%$. É comum para compósitos reforçados com celulose o aumento do módulo elástico, uma vez que a celulose é um material de alta cristalinidade que pode agir como entrecruzador físico, melhorando a rigidez do material [15, 20-23]. O aumento no módulo elástico é um forte indicativo que a nanocelulose está dispensa de forma uniforme na matriz polimérica e que a adesão interfacial é garantida pela formação de ligações de hidrogênio entre os grupos hidroxilas da celulose e os grupos aminas da matriz polimérica $[8,9]$. Com a adição de 2\% de NFC (amostra PA-P2) o aumento foi menor, apenas 5\%. As amostras pré-misturadas tiveram uma diminuição na deformação plástica e deformação máxima de $4,4 \pm$ 0,2 \% (amostra PA-P0) para 3,3 \pm 0,3 \% (amostra PA-P1) e 3,8 \pm 0,4 \% (amostra PA-P2), correspondendo a uma redução de $10 \%$, em média. Esse decréscimo na ductibilidade com a adição de fibras em polímeros é uma tendência comumente observada para compósitos termoplásticos [9, 20, 24, 25].

Tabela 3: Propriedades mecânicas das amostras.

\begin{tabular}{l|l|l|l}
\hline AMOSTRA & $\begin{array}{l}\text { MÓDULO ELÁSTICO } \\
(\mathrm{GPa})\end{array}$ & $\begin{array}{l}\text { RESISTÊNCIA À } \\
\text { TRAÇÃO (MPa) }\end{array}$ & $\begin{array}{l}\text { DEFORMAÇÃO NA } \\
\text { RUPTURA (\%) }\end{array}$ \\
\hline
\end{tabular}




\begin{tabular}{l|l|l|l}
\hline PA-0 & $1,9 \pm 0,1$ & $64,5 \pm 1,0$ & $4,9 \pm 0,1$ \\
\hline PA-P0 & $1,9 \pm 0,1$ & $64,0 \pm 1,0$ & $4,4 \pm 0,2$ \\
\hline PA-P1 & $2,2 \pm 0,1$ & $61,0 \pm 0,8$ & $3,3 \pm 0,3$ \\
\hline PA-P2 & $2,0 \pm 0,2$ & $54,0 \pm 5,0$ & $3,8 \pm 0,4$ \\
\hline
\end{tabular}

\section{CONCLUSÕES}

A partir das caracterizações realizadas com a NFC foi possível observar sua alta estabilidade térmica, estando isso de acordo com sua alta cristalinidade. Quanto maior é a temperatura de início de decomposição, melhor é a estabilidade térmica dessas fibras em comparação com outras fibras naturais, indicando uma suscetibilidade para o processamento com termoplásticos, mesmo utilizando temperatura de fusão elevada. A poliamida 6,6 utilizada neste estudo apresentou alta estabilidade térmica $\left(\mathrm{T}_{\mathrm{i}}=380^{\circ} \mathrm{C}\right)$, como esperado. A pré-mistura resultou em material homogêneo, sem alteração de coloração e de fácil obtenção. Durante o processamento por extrusão e injeção houve o grande desafio de trabalhar no limite de temperatura de fusão da PA antes que as NFC começassem a degradar. Entretanto, foi possível a obtenção de corpos de prova homogêneos, com uma leve coloração para os que continham NFC, indicando a influência das mesmas na estabilidade térmica dos materiais finais. Os resultados de TG confirmaram a menor estabilidade térmica das amostras com NFC em relação às amostras sem NFC. Para as amostras sem NFC pré-misturadas ou não, a estabilidade térmica também foi menor em comparação com os pellets do polímero original, mostrando a influência da prémistura e do próprio processamento. Os resultados dos ensaios mecânicos para essas amostras revelaram que, além da pré-mistura não ter prejudicado o módulo elástico e a resistência à tração, a adição de NFC levou a um aumento de $16 \%$ do módulo elástico. A redução da deformação máxima observada para as amostras com NFC é comum em materiais mais rígidos. Houve essa redução mesmo para a amostra sem NFC (PA-P0), indicando novamente a influência da pré-mistura nas propriedades finais do material. Com os resultados apresentados, podemos concluir que tanto a pré-mistura como a extrusão, utilizados para dispersar e incorporar as NFC, apesar de não melhorarem expressivamente as propriedades mecânicas dos materiais finais, não prejudicaram o módulo elástico e a resistência máxima à tração, podendo indicar uma alternativa de processamento de nanocompósitos de poliamida 6,6 reciclada e NFC, dependendo da aplicação final do material.

\section{AGRADECIMENTOS}

Ao CNPq (309779/2010-3) e a CAPES (155514/2011-3) pelo apoio financeiro. Ao Prof. M. Sain pela doação das NFC e a empresa Rhodia pela doação da resina de PA. A Prof. M. C. Gonçalves pelo uso da miniextrusora e injetora. Ao técnico R. G. Pereira pelo apoio nos laboratórios.

\section{BIBLIOGRAFIA}

[1] KHALIL, H. P. S. A., BHAT, A. H., YUSRA, A. F. I. "Green composites from sustainable cellulose nanofibrils: A review", Carbohydrate Polymers, v. 87, pp. 963-979, 2012.

[2] RETO, M. A. S. Plásticos Especiais: Montadoras movem novos desenvolvimentos na indústria de resina. Disponível em: <http://www.plastico.com.br/plastico/plastico/plasticos-especiais-montadoras-movem-novosdesenvolvimentos-na-industria-de-resina/>, 2012.

[3] SAMIR, M. A. S. A., ALLOIN, F., DUFRESNE, A. "Review of recent research into cellulosic whiskers, their properties and their application in nanocomposite field", Biomacromolecules, v. 6, pp. 612-626, 2005.

[4] KIZILTAS, A., GARDNER, D. J., HAN, Y., et al., "Dynamic mechanical behavior and thermal properties of microcrystalline cellulose (MCC)-filled nylon 6 composites", Thermochimica Acta, v. 519, pp. 38-43, 2011.

[5] ALI, I., ELLEITHY, R. "Toughness of $\mathrm{HDPE} / \mathrm{CaCO}_{3}$ microcomposites prepared from masterbatch by melt blend method", Journal of Applied Polymer Science, v. 122, pp. 3303-3315, 2011.

[6] JOHN, M. J., THOMAS, S. "Biofibres and biocomposites: Review", Carbohydrate Polymers, v. 71, pp. 343-364, 2008.

[7] SIQUEIRA, G., BRAS, J., DUFRESNE, A. "Cellulose whiskers versus microfibrils: influence of the nature of the nanoparticle and its surface functionalization on the thermal and mechanical properties of nanocomposites", Biomacromolecules, v. 10, pp. 425-432, 2009. 
[8] QUA, E. H., HORNSBY, P. R. "Preparation and characterization of nanocellulose reinforced polyamide6”. Plastics, Rubber and Composites, v. 40(6/7), pp. 300-306, 2011.

[9] CORREAA, A. C., TEIXEIRA, E. M., CARMONA, V. B., et al., "Obtaining nanocomposites of polyamide 6 and cellulose whiskers via extrusion and injection molding”, Cellulose, v. 21, pp. 311-322, 2014.

[10] DURÁN, N., MATTOSO, L. H. C., MORAIS, P. C. Nanotecnologia: introdução, preparação e caracterização de nanomateriais e exemplos de aplicação, São Paulo, Artliber, 2006.

[11] MANTIA, F. P. L., MORREALE, M., SCAFFARO, R., et al., "Rheological and Mechanical Behavior of LDPE/Calcium Carbonate Nanocomposites and Microcomposites", Journal of Applied Polymer Science, v. 127, pp. 2544-2552, 2013.

[12] RODIONOVA, G., LENES, M. "Surface chemical modification of microfibrillated cellulose: improvement of barrier properties for packaging applications", Cellulose, v. 18, pp. 127-134, 2011.

[13] CHANDA, M., ROY, S. K. Industrial polymers, specialty polymers, and their applications, Boca Raton, Taylor \& Francis Group, 2009.

[14] KIZILTAS, A., NAZARI, B., GARDNER, D. J., et al., "Polyamide 6-cellulose composites: effect of cellulose composition on melt rheology and crystallization behavior", Polymer Engineering and Science, v. 54, pp. 739-756, 2014.

[15] ALEMDAR, A., SAIN, M. "Biocomposites from wheat straw nanofibers: Morphology, thermal and mechanical properties", Composites Science and Technology, v. 68, pp. 557-565, 2008.

[16] ALEMDAR, A., SAIN, M. "Isolation and characterization of nanofibers from agricultural residues Wheat straw and soy hulls", Bioresource Technology, v. 99, pp. 1664-1671, 2008.

[17] BENADUCCI, D. "Nanocompósitos de poliamida 6,6 reciclada reforçados com nanofibras de celulose para aplicação em peças automotivas”, Dissertação de M.Sc, Escola de Engenharia de São Carlos, Universidade de São Paulo, 2014.

[18] PANTHAPULAKKAL, S., SAIN, M. "Preparation and characterization of cellulose nanofibril films from wood fibre and their thermoplastic polycarbonate composites", International Journal of Polymer Science, v. 2012, pp. 1-6, 2012.

[19] PALMER, R. J. Polyamides, Plastics. In Encyclopedia of Polymer Science and Technology 3. John Wiley \& Sons, 2001.

[20] IWATAKE, A., NOGI, M., YANO, H. "Cellulose nanofiber-reinforced polylactic acid", Composites Science and Technology, v. 68, pp. 2103-2106, 2008.

[21] JONOOBI, M., HARUR, J., MATHEW, A. P., et al., "Mechanical properties of cellulose nanofiber (CNF) reinforced polylactic acid (PLA) prepared by twin screw extrusion", Composites Science and Technology, v. 70, pp. 1742-1747, 2010.

[22] LU, J., WANG, T., DRZAL, L. T. "Preparation and properties of microfibrillated cellulose polyvinyl alcohol composite materials", Composites: Part A, v. 39, pp. 738-746, 2008.

[23] MATHEW, A. P., OKSMAN, K., SAIN, M. "Mechanical properties of biodegradable composites from poly lactic acid (PLA) and microcrystalline cellulose (MCC)", Journal of Applied Polymer Science, v. 97, pp. 2014-2025, 2005.

[24] SALEHI-MOBARAKEH, H., YADEGARI, A., KHAKZAD-ESFAHLAN, F., et al., "Modifying montmorillonite clay via silane grafting and interfacial polycondensation for melt compounding of nylon-66 nanocomposite", Journal of Applied Polymer Science, v. 124, pp. 1501-1510, 2012.

[25] SOUZA, S. F., LEÃO, A. L., CAI, J. H., et al. "Nanocellulose from Curaua Fibers and their Nanocomposites", Molecular Crystals and Liquid Crystals, v. 522, pp. 342-352, 2010. 